\title{
RESPOSTA DE Pinus taeda COM DIFERENTES IDADES À ADUBAÇÃO NPK NO PLANALTO SUL CATARINENSE ${ }^{(1)}$
}

\author{
Letícia Moro $^{(2)}$, Luciano Colpo Gatiboni ${ }^{(3)}$, Marcia Aparecida Simonete ${ }^{(4)}$, Paulo Cezar \\ Cassol $^{(3)}$ \& Djalma Miler Chaves ${ }^{(5)}$
}

\begin{abstract}
RESUMO
Na região do Planalto Sul-catarinense, a maioria das florestas de Pinus foi implantada sem fertilização do solo na fase de plantio. Atualmente, muitas áreas encontram-se em segunda ou terceira rotação de Pinus e sem nenhuma fertilização, o que pode limitar a produtividade pela baixa fertilidade do solo. Uma alternativa para a mitigação desse problema seria a adubação em povoamentos já estabelecidos. Com o objetivo de avaliar o efeito da adubação com N, P e K, em diferentes fases de crescimento de Pinus taeda, foram conduzidos experimentos no campo, em plantios de um, cinco e nove anos de idade, todos de segunda rotação, sobre Cambissolos no município de Otacílio Costa, SC. Os tratamentos consistiram de combinações de doses de $\mathrm{N}\left(\mathrm{N} 0=0, \mathrm{~N} 1=70\right.$ e N2 $\left.=140 \mathrm{~kg} \mathrm{ha}^{-1} \mathrm{de} \mathrm{N}\right)$, $\mathrm{P}\left(\mathrm{P0}=0, \mathrm{P} 1=75\right.$ e P2 $\left.=150 \mathrm{~kg} \mathrm{ha}^{-1} \mathrm{P}_{2} \mathrm{O}_{5}\right)$ e K $\left(\mathrm{Ko}=0, \mathrm{~K} 1=60\right.$ e K2 $=120 \mathrm{~kg} \mathrm{ha}^{-1} \mathrm{de}$ $\mathrm{K}_{2} \mathrm{O}$ ), além de uma testemunha, nas seguintes combinações: N0P0K0, NOP1K0, N1P1K1, N1P2K1, N1P2K2 e N2P2K1. O delineamento experimental utilizado foi de blocos ao acaso com três repetições. Foram avaliados altura e diâmetro no colo ou à altura do peito das árvores e calculado o volume de tronco das plantas e teores de N, P e K nas acículas aos seis e 18 meses, após a aplicação dos tratamentos. Os resultados evidenciaram que nas plantas de um ano de idade houve incremento significativo no volume de madeira com a aplicação de $70 \mathrm{~kg} \mathrm{ha}^{-1}$ de $\mathrm{P}_{2} \mathrm{O}_{5}$. Para os plantios de cinco e nove anos, a adição de doses a partir de 70,75 e $60 \mathrm{~kg} \mathrm{ha}^{-1} \mathrm{de} \mathrm{N}$, $\mathrm{P}_{2} \mathrm{O}_{5}$ e $\mathrm{K}_{2} \mathrm{O}$, respectivamente, promoveram incrementos significativos no volume de madeira, mostrando que a adubação em florestas já estabelecida é uma estratégia a ser considerada em sítios de baixa fertilidade.
\end{abstract}

Termos de indexação: nutriente, época de aplicação, produção.

(1) Parte da Dissertação de Mestrado do primeiro autor, junto à Universidade do Estado de Santa Catarina - UDESC. Recebido para publicação em 4 de setembro de 2013 e aprovado em 6 de maio de 2014.

(2) Doutoranda em Ciência do Solo, Centro de Ciências Agroveterinárias, UDESC. Av. Luis de Camões, 2090. CEP 88520-000 Lages (SC). E-mail: leticia_moro@hotmail.com

(3) Professor, Departamento de Solos, UDESC. Bolsista de produtividade em pesquisa do CNPq. E-mail: lgatiboni@gmail.com, a2pcc@cav.udesc.br

(4) Pós-Doutoranda em Ciência do solo, Departamento de Solos, UDESC. E-mail: maapasi@ig.com.br

(5) Consultor da Klabin S.A.. Fazenda Monte Alegre, s/n. CEP 84279-000 Telêmaco Borba (PR). E-mail: dm.chaves@terra.com.br 


\title{
SUMMARY: RESPONSE OF ONE-, FIVE-, AND NINE-YEAR-OLD Pinus taeda TO NPK FERTILIZATION IN SOUTHERN BRAZIL
}

\begin{abstract}
In the southern highland (Planalto Sul) region of Santa Catarina, Brazil, most of the pine forests were planted without soil fertilization. Currently, many areas are in the second or third rotation, and their productivity may be limited by low soil fertility. An alternative to mitigate this problem would be fertilization in the established forest. This study was carried out with the objective of evaluating the effect of fertilization with $N, P$, and $K$ at different growth stages of Pinus taeda. Experiments were conducted in one-, five-, and nine-year-old forests, all in the second rotation, in Cambisols located in the municipality of Otacilio Costa, Santa Catarina, Brazil. Treatments consisted of combinations of fertilization rates of $N(N O=0, N 1=70$, and $\left.N 2=140 \mathrm{~kg} \mathrm{ha}^{-1} \mathrm{~N}\right), P\left(P 0=0, P 1=75\right.$, and $\left.P 2=150 \mathrm{~kg} \mathrm{ha}^{-1} P_{2} \mathrm{O}_{5}\right)$, and $K(K 0=0, K 1=60$, and $\left.K 2=120 \mathrm{~kg} \mathrm{ha}^{-1} \mathrm{~K}_{2} \mathrm{O}\right)$, plus a control treatment without fertilization. The combinations used were NOP0K0, NOP1K0, N1P1K1, N1P2K1, N1P2K2, and N2P2K1. A completely randomized block experimental design was used, with three replications. The height and diameter at the root collar or at breast height of trees was measured; also the trunk volume of the plants and N, $P$, and $K$ contents in needles at six and 18 months after fertilization were measured. In one-year-old plants, a significant increase in timber volume was obtained with the application of $70 \mathrm{~kg} \mathrm{ha}^{-1}$ of $\mathrm{P}_{2} \mathrm{O}_{5}$. For five- and nine-year-old plants, fertilization with 70, 75, and $60 \mathrm{~kg} \mathrm{ha}^{-1}$ of $\mathrm{N}, \mathrm{P}_{2} \mathrm{O}_{5}$, and $\mathrm{K}_{2} \mathrm{O}$, respectively, promote significant increase in timber volume, showing that fertilization of established pine forests increase productivity in areas of low fertility.
\end{abstract}

Index terms: nutrients, application times, productivity.

\section{INTRODUÇÃO}

No Brasil, a maioria das florestas de Pinus taeda tem sido implantada sem fertilização de plantio, enquanto nos Estados Unidos da América (EUA) a aplicação de fertilizantes tornou-se prática comum para aumentar a área foliar e produção de lenho (Albaugh et al., 2007). Em razão das condições edafoclimáticas do território brasileiro, as florestas de Pinus apresentam rápido crescimento e incremento médio anual (IMA) de $25 \mathrm{~m}^{3} \mathrm{ha}^{-1} \mathrm{ano}^{-1}$, considerado excelente em comparação com a média nos EUA, onde a cultura é nativa e apresenta IMA de apenas 10 $\mathrm{m}^{3} \mathrm{ha}^{-1}$ ano $^{-1}$, evidenciando a adaptação da espécie no território brasileiro (Juvenal \& Mattos, 2002). Pinto Jr. et al. (2013) relataram casos de plantações em sítios especiais cujo IMA chega a alcançar $45 \mathrm{~m}^{3} \mathrm{ha}^{-1} \mathrm{ano}^{-1}$ ou mais.

As espécies do gênero Pinus apresentam uma excepcional capacidade de utilização dos recursos nutricionais em solos de baixa fertilidade, com rápido crescimento, sem apresentar sintomas visuais de deficiência, dando a falsa expectativa de que nos plantios não seriam necessários grandes cuidados com a fertilização (Reissmann \& Wisniewski, 2005). Porém, plantas crescidas nessas condições certamente não apresentam desenvolvimento economicamente satisfatório (Reissmann \& Wisniewski, 2005; Viera \& Schumacher, 2009). Essa condição, associada ao fato da não fertilização dos plantios, à exportação de nutrientes nos desbastes e na colheita final e às perdas por processos erosivos e de lixiviação, leva a uma aceleração no empobrecimento dos níveis de fertilidade química do solo e nas perdas de produtividades futuras dos plantios.
Segundo Ferreira et al. (2001), os trabalhos desenvolvidos no Brasil concluem que o Pinus, de forma geral, responde menos à adubação que o Eucalyptus, mas que incrementos em volume podem chegar a $20 \%$ ou mais em solos com baixa fertilidade, e que melhores resultados foram obtidos com a aplicação de $\mathrm{P}, \mathrm{K}$ e calcário como fonte de $\mathrm{Ca}$ e Mg.

Vários estudos na América do Sul e no sul dos EUA têm relatado aumentos no crescimento de Pinus taeda em razão da fertilização no plantio (Fernández et al., 1999; 2000; Bekele et al., 2003; Ibañez et al., 2004; Vogel et al., 2005; Fox et al., 2007; Everett \& PalmLeis, 2009; Faustino et al., 2011; 2013). Contudo, os estudos sobre as árvores jovens e de curto prazo não são adequados para determinar a absorção de nutrientes ao longo do tempo, pois essas podem não atingir o crescimento e as taxas de absorção comparáveis com os dos plantios mais maduros (Bekele et al., 2003). Além disso, não necessariamente elementos que limitam a rotação no início são também limitantes em longo prazo (Fox et al., 2007).

Segundo Jokela \& Long (2000), os requisitos para fertilização de plantios estabelecidos devem ser com base nos mesmos princípios da adubação no plantio. No entanto, as pesquisas são necessárias, pois é mais difícil de prever a necessidade de fertilizantes em plantios mais velhos, porque a penetração das raízes profundas pode permitir absorção de nutrientes dos horizontes do subsolo, além de camadas superficiais de detritos orgânicos, por exemplo, acículas, também servirem como reservatório de nutrientes, liberando nutrientes pela lenta decomposição desse material.

Para definir as idades de aplicação dos fertilizantes, é imprescindível considerar as fases de crescimento da floresta. Durante a fase de estabelecimento, que 
ocorre até o primeiro ano após o plantio, a fertilização é importante porque o sistema radicular ainda não está totalmente desenvolvido, sofrendo com a competição das plantas espontâneas, os ataques de pragas, as variações climáticas e o tipo de preparo do solo (Flinn, 1985). Com isso, reduziria a competição por nutrientes do solo entre o Pinus e as plantas de sub-bosque e aumentaria o desenvolvimento da folhagem, contribuindo para maior interceptação da luz solar (Jokela, 2004) e sofrendo menos com as adversidades do ambiente.

Por volta dos seis anos, ocorre o maior crescimento de madeira juvenil. Clark III \& Saucier (1991) observaram que os nutrientes são fator limitante no crescimento de madeira juvenil. É também o período em que se inicia o fechamento do dossel, exigindo grande quantidade de nutrientes. Jokela et al. (1991) relataram que nesse período as condições ambientais não são tão propícias à alta disponibilidade de nutrientes, pois o fornecimento de nutrientes pela serapilheira não é suficiente, o que pode resultar em um desenvolvimento de área foliar restrito.

Aproximadamente aos nove anos, passa a ocorrer competição por luz, espaço e nutrientes; há redução gradativa na expansão da copa, passando a formar madeira adulta; inicia a transição entre lenho juvenil e adulto (Clark III \& Saucier, 1991); há a transformação do alburno em cerne (Foelkel, 1976), demandando grande quantidade de nutrientes e ocorrendo concomitantemente o acúmulo máximo de nutrientes (Jokela et al., 1991). Nesse período, geralmente os processos de decomposição abrandam e a disponibilidade de nutrientes diminui, pois esses são imobilizados no tronco, na casca, nos ramos, nas raízes e na folhagem (Jokela, 2004); se a nutrição não for adequada, acículas podem ser perdidas prematuramente.

Vários trabalhos com adubação em plantios já estabelecidos desenvolvidos nos EUA têm demostrando efeitos positivos sobre o crescimento (Kyle et al., 2005; Will et al., 2006; Albaugh et al., 2008). Entretanto, no Brasil, esses estudos são escassos e relativamente antigos, tendo em vista o desenvolvimento do melhoramento genético desde então.

Diante do exposto, este trabalho teve por objetivo avaliar o efeito da aplicação de doses de N, P e K no crescimento de plantios de Pinus taeda, com um, cinco e nove anos de idade, no Planalto Sul-catarinense.

\section{MATERIAL E MÉTODOS}

Os experimentos foram instalados em plantios de Pinus taeda L. com um, cinco e nove anos de idade, implantados em 2009, 2005 e 2001, respectivamente, em áreas da empresa Klabin S.A., no município de Otacílio Costa, SC. O plantio de um ano encontra-se na Fazenda Bom Retiro; e os de cinco e nove anos, na
Fazenda Cervo, com as respectivas coordenadas geográficas: $58^{\circ} 68^{\prime} 05^{\prime \prime} \mathrm{S}$ e $97^{\circ} 19^{\prime} 39^{\prime \prime} \mathrm{W} ; 59^{\circ} 09^{\prime} 46^{\prime \prime} \mathrm{S}$ e $96^{\circ} 26^{\prime} 45^{\prime \prime} \mathrm{W}$; e $58^{\circ} 95^{\prime} 54^{\prime \prime} \mathrm{S}$ e $96^{\circ} 30^{\prime} 08^{\prime \prime} \mathrm{W}$. O clima da região é mesotérmico úmido com verão ameno, Cfb, segundo a classificação de Köppen. A altitude é de $884 \mathrm{~m}$, a temperatura média anual é de $16{ }^{\circ} \mathrm{C}$ e a precipitação pluvial média anual de $1.500 \mathrm{~mm}$, bem distribuída ao longo do ano (Santa Catarina, 2012).

No plantio de um ano, o solo é Cambissolo Háplico alumínico típico, e nos plantios de cinco e nove anos, Cambissolo Húmico alumínico típico, cujas principais características são apresentadas no quadro 1. As áreas experimentais encontram-se no segundo ciclo de plantio, cujo ciclo anterior também era de Pinus taeda L. Todos os plantios foram efetuados após o preparo do solo com subsolagem, utilizando trator de esteira, na profundidade em torno de $45 \mathrm{~cm}$, e em espaçamento de $2,5 \mathrm{~m}$ entre plantas e $2,5 \mathrm{~m}$ entrelinhas, sem adubação no plantio; o plantio foi em sulcos.

Os tratamentos consistiram da combinação de doses de $\mathrm{N}: \mathrm{N} 0=0, \mathrm{~N} 1=70$ e N2 $=140 \mathrm{~kg} \mathrm{ha}^{-1}$ de N; $\mathrm{P}: \mathrm{P} 0=0, \mathrm{P} 1=75$ e $\mathrm{P} 2=150 \mathrm{~kg} \mathrm{ha}^{-1} \mathrm{de}_{2} \mathrm{O}_{5}$; e K: $\mathrm{K} 0=0, \mathrm{~K} 1=60$ e $\mathrm{K} 2=120 \mathrm{~kg} \mathrm{ha}^{-1}$ de $\mathrm{K}_{2} \mathrm{O}$, ficando nomeados da seguinte forma: NOP0K0, NOP1K0, N1P1K1, N1P2K1, N1P2K2 e N2P2K1. Como fontes dos nutrientes, foram usados ureia ( $45 \%$ de $\mathrm{N})$, superfosfato triplo $\left(41 \%\right.$ de $\left.\mathrm{P}_{2} \mathrm{O}_{5}\right)$ e cloreto de potássio (58\% de $\mathrm{K}_{2} \mathrm{O}$ ).

O delineamento experimental utilizado foi de blocos ao acaso com três repetições, sendo as parcelas compostas por oito linhas contendo 10 plantas cada, totalizando 80 plantas por parcela, das quais somente as 48 plantas centrais (seis linhas $\times$ oito plantas) foram utilizadas para as mensurações dendrométricas; e 24 plantas centrais (quatro linhas $\times$ seis plantas), para coletas de acículas.

Em dezembro de 2010, antes da aplicação dos tratamentos, foram coletadas amostras de solo e acículas das árvores, bem como realizadas mensurações dendrométricas. Em 21 de dezembro, procedeu-se à aplicação dos tratamentos, em que os fertilizantes foram aplicados a lanço e em área total. $\mathrm{E}$, aos seis e 18 meses após a aplicação dos tratamentos (junho/2011 e junho/2012, respectivamente), foram novamente realizadas amostragem de acículas e mensurações dendrométricas.

Para fins de caracterização, o solo foi coletado nas profundidades de 0-20 e 20-40 cm, com trado holandês, em cinco pontos dentro de cada parcela. As amostras, após serem secas em estufa de circulação de ar forçada a $60^{\circ} \mathrm{C}$, por $48 \mathrm{~h}$, foram moídas e submetidas à análise química segundo Tedesco et al. (1995) (Quadro 1).

A amostragem de tecido vegetal foi efetuada retirando-se três fascículos de cada ponto cardeal no terço superior da copa, no antepenúltimo lançamento das folhas dos galhos. Os fascículos foram secos a $65{ }^{\circ} \mathrm{C}$ em estufa com circulação de ar forçada, até atingir massa constante. Posteriormente, as amostras 
Quadro 1. Características químicas dos Cambissolos nas áreas experimentais das floretas de Pinus taeda

\begin{tabular}{|c|c|c|c|c|c|c|c|c|c|c|c|}
\hline Camada & $\mathrm{pH}\left(\mathrm{H}_{2} \mathrm{O}\right)$ & Argila & MO & $\mathbf{P}$ & $\mathbf{K}$ & $\mathbf{C a}$ & Mg & Al & $\mathrm{H}+\mathrm{Al}$ & $\mathrm{CTC}_{\mathrm{pH} 7}$ & $\mathbf{m}^{(1)}$ \\
\hline \multirow[t]{2}{*}{$\mathrm{cm}$} & & - dą & 1 - & $-\mathrm{m}$ & 3 & 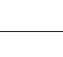 & 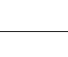 & $\mathrm{nol}_{\mathrm{c}} \mathrm{dn}$ & & & $\%$ \\
\hline & \multicolumn{11}{|c|}{ Cambissolo Háplico alumínico típico (plantio de um ano) } \\
\hline $0-20$ & 4,1 & 17,7 & 5,8 & 5,1 & 72,2 & 0,2 & 0,2 & 10,0 & 38,4 & 39,0 & 94,4 \\
\hline \multirow[t]{2}{*}{$20-40$} & 4,2 & 25,6 & 3,7 & 2,1 & 37,7 & 0,1 & 0,1 & 8,9 & 33,9 & 34,2 & 96,3 \\
\hline & \multicolumn{11}{|c|}{ Cambissolo Húmico alumínico típico (plantio de cinco anos) } \\
\hline $0-20$ & 4,0 & 23,2 & 6,2 & 5,0 & 65,1 & 0,3 & 0,3 & 10,9 & 41,8 & 42,5 & 93,4 \\
\hline \multirow[t]{2}{*}{$20-40$} & 4,2 & 33,8 & 4,3 & 2,5 & 47,7 & 0,2 & 0,2 & 10,7 & 37,8 & 38,4 & 94,6 \\
\hline & \multicolumn{11}{|c|}{ Cambissolo Húmico alumínico típico (plantio de nove anos) } \\
\hline $0-20$ & 4,1 & 22,3 & 5,6 & 4,3 & 29,6 & 0,2 & 0,1 & 6,9 & 33,9 & 34,2 & 94,9 \\
\hline $20-40$ & 4,2 & 32,9 & 4,1 & 1,9 & 16,8 & 0,2 & 0,1 & 6,0 & 31,0 & 31,3 & 95,0 \\
\hline
\end{tabular}

(1) Saturação por alumínio na $\mathrm{CTC}_{\text {efetiva. }}$

foram moídas e submetidas à digestão com ácido sulfúrico e água oxigenada, sendo as concentrações de $\mathrm{N}$ e $\mathrm{K}$ determinadas de acordo com descrito por Tedesco et al. (1995), e o P com proposto por Murphy \& Riley (1962).

As mensurações dendométricas efetuadas foram: circunferência à altura do colo - CAC (plantio de um ano), circunferência a altura do peito - CAP (plantios de cinco e nove anos) e altura - $\mathrm{H}$ (nos três plantios). A mensuração do CAC (plantio de um ano) e a do CAP (plantios de cinco e nove anos) foram realizadas com fita métrica, e a altura $(\mathrm{H})$ realizou-se com régua (plantio de um ano) e aparelho Hipsômetro Vertex (plantios de cinco e nove anos).

Os dados de CAC e CAP foram convertidos para diâmetro à altura do colo (DAC) e diâmetro à altura do peito (DAP), respectivamente. Calculou-se o volume médio individual de tronco $(v)$ de cada tratamento.

Para cálculo de $v$ no plantio de um ano, utilizou-se a seguinte equação:

$$
v=h\left[\left(\pi D A C^{2}\right) / 4\right] f
$$

Enquanto para os plantios de cinco e nove anos, utilizou-se a equação de Schumacher-Hall Logarítmica:

$$
\ln v=b_{0}+b_{1} \ln d+b_{2} \ln
$$

em que $v=$ volume médio individual, $\mathrm{em}^{3} ; D A C=$ diâmetro à altura do colo, em $\mathrm{m}$; $d=$ diâmetro à altura do peito, em $\mathrm{cm} ; h=$ altura total, em $\mathrm{m} ; b_{0}, b_{1}$ e $b_{2}=$ parâmetros; $f=$ fator de forma; e $l n=$ logaritmo neperiano.

O volume de madeira por hectare foi calculado usando os seguintes stands observados nos três florestamentos: 1533; 1367; e 1.400 árvores ha ${ }^{-1}$, para os respectivos plantios de um, cinco e nove anos.

Foram calculados aos 18 meses os incrementos correntes em DAC, DAP, $\mathrm{H}, \mathrm{V}$, em $\mathrm{m}^{3}$ /árvore, e V, $\mathrm{m}^{3} \mathrm{ha}^{-1}$, o incremento corrente anual em DAC, DAP, $\mathrm{H} \mathrm{e} \mathrm{V}, \mathrm{em} \mathrm{m}^{3} \mathrm{ha}^{-1} \mathrm{e}$ o incremento médio anual em V, em $\mathrm{m}^{3} \mathrm{ha}^{-1}$, conforme equações:
Incremento Corrente Anual $(I C A)=$ medição aos 18 meses - medição inicial/1,5

Incremento Médio Anual (IMA) =

medição aos 18 meses/idade do plantio

Os dados foram submetidos à análise de variância pelo teste $\mathrm{F}$, e as médias, comparadas pelo Teste de Scott-Knott, a $5 \%$. Para tanto, utilizou-se o programa estatístico R (R Development Core Team, 2011).

\section{RESULTADOS E DISCUSSÃO}

O incremento corrente anual (ICA) expressa o crescimento ocorrido em um período de 12 meses, correspondendo o que a árvore cresceu no período de um ano. $\mathrm{O}$ valor do incremento médio anual (IMA) expressa a média do crescimento total à certa idade da árvore; portanto, expressa a média anual do crescimento para qualquer idade (Encinas et al., 2005).

Houve efeito significativo da aplicação de N, P e $\mathrm{K}$, na maioria das diferentes combinações e doses, no ICA de diâmetro à altura do colo ou peito (ICA-DAC ou ICA-DAP), à altura (ICA-H) e ao volume de tronco (ICA-V) para os três plantios (Quadro 2).

No plantio fertilizado com um ano, todos os tratamentos que receberam um ou mais nutrientes nas diferentes doses apresentaram maior ICA em diâmetro e em altura, proporcionando maior ICA de volume de tronco das árvores em relação à testemunha; entretanto, quando comparados entre si, não demonstraram (Quadro 2). Os incrementos foram de $5,6 \%$ para ICA em altura, variando de 13,8 a $20,7 \%$ para ICA em diâmetro, e de 22,3 a $36,7 \%$ para ICA em volume. Isto demonstra que a aplicação de $\mathrm{P}$, a partir de $75 \mathrm{~kg} \mathrm{ha}^{-1}$, combinado ou não com $\mathrm{N}$ e K, na fase inicial de desenvolvimento do Pinus taeda proporciona a formação de árvores de maior espessura e altura, aspectos que podem refletir futuramente na produção. 
Quadro 2. Incremento corrente anual (ICA) de diâmetro à altura do colo (DAC), diâmetro à altura do peito (DAP), da altura (H) e do volume de tronco (V), avaliados aos 18 meses após a aplicação dos tratamentos, em florestas de Pinus taeda com idades de um, cinco e nove anos

\begin{tabular}{|c|c|c|c|c|c|c|c|c|c|}
\hline \multirow{2}{*}{ Tratamento $^{(1)}$} & \multicolumn{3}{|c|}{ Um ano } & \multicolumn{3}{|c|}{ Cinco anos } & \multicolumn{3}{|c|}{ Nove anos } \\
\hline & ICA-DAC & ICA-H & ICA-V & ICA-DAP & ICA-H & ICA-V & ICA-DAP & ICA-H & ICA-V \\
\hline & $\mathrm{cm}$ & $\mathrm{m}$ & $\mathrm{m}^{3} \mathrm{ha}^{-1}$ & $\mathrm{~cm}$ & $\mathrm{~m}$ & $\mathrm{~m}^{3} \mathrm{ha}^{-1}$ & $\mathrm{~cm}$ & $\mathrm{~m}$ & $\mathrm{~m}^{3} \mathrm{ha}^{-1}$ \\
\hline NOP0K0 & $2,9 \mathrm{~b}$ & $1,8 \mathrm{~b}$ & $16,6 \mathrm{~b}$ & $2,5 \mathrm{~b}$ & $2,5 \mathrm{~b}$ & $62,5 \mathrm{~b}$ & $0,8 \mathrm{~b}$ & $1,7 \mathrm{c}$ & $63,4 \mathrm{~b}$ \\
\hline N0P1K0 & $3,4 \mathrm{a}$ & $1,9 \mathrm{a}$ & $20,4 \mathrm{a}$ & $2,7 \mathrm{a}$ & $2,4 \mathrm{c}$ & $65,7 \mathrm{~b}$ & $0,9 \mathrm{~b}$ & $2,0 \mathrm{~b}$ & $77,7 \mathrm{~b}$ \\
\hline $\mathrm{N} 1 \mathrm{P} 1 \mathrm{~K} 1$ & 3,5 a & $1,9 \mathrm{a}$ & $22,7 \mathrm{a}$ & $2,7 \mathrm{a}$ & $2,7 \mathrm{a}$ & $68,7 \mathrm{a}$ & $0,9 \mathrm{~b}$ & $1,8 \mathrm{~b}$ & $75,2 \mathrm{~b}$ \\
\hline N1P2K1 & $3,4 \mathrm{a}$ & $1,9 \mathrm{a}$ & $21,0 \mathrm{a}$ & $2,8 \mathrm{a}$ & $2,7 \mathrm{a}$ & $71,2 \mathrm{a}$ & $1,0 \mathrm{~b}$ & $2,1 \mathrm{a}$ & $83,2 \mathrm{a}$ \\
\hline $\mathrm{N} 1 \mathrm{P} 2 \mathrm{~K} 2$ & $3,4 \mathrm{a}$ & $1,9 \mathrm{a}$ & $20,7 \mathrm{a}$ & $2,5 \mathrm{~b}$ & $2,3 \mathrm{c}$ & $61,6 \mathrm{~b}$ & $1,2 \mathrm{a}$ & $2,2 \mathrm{a}$ & $89,2 \mathrm{a}$ \\
\hline $\mathrm{N} 2 \mathrm{P} 2 \mathrm{~K} 1$ & $3,3 \mathrm{a}$ & $1,9 \mathrm{a}$ & $20,3 \mathrm{a}$ & $2,8 \mathrm{a}$ & $2,8 \mathrm{a}$ & 71,9 a & $0,9 \mathrm{~b}$ & $1,8 \mathrm{~b}$ & $71,0 \mathrm{~b}$ \\
\hline CV (\%) & 15,1 & 8,0 & 27,6 & 17,2 & 9,0 & 20,1 & 32,8 & 14,0 & 31,6 \\
\hline
\end{tabular}

(1) $\mathrm{N} 0 \mathrm{P} 0 \mathrm{~K} 0=0 \mathrm{~kg} \mathrm{ha}^{-1}$ de $\mathrm{N}, \mathrm{P}_{2} \mathrm{O}_{5}$ e $\mathrm{K}_{2} \mathrm{O} ; \mathrm{N} 0 \mathrm{P} 1 \mathrm{~K} 0=0,75$ e $0 \mathrm{~kg} \mathrm{ha}^{-1}$ de $\mathrm{N}, \mathrm{P}_{2} \mathrm{O}_{5}$ e $\mathrm{K}_{2} \mathrm{O}$, respectivamente; N1P1K1 = 70, 75 e $60 \mathrm{~kg} \mathrm{ha}^{-1}$ de N, $\mathrm{P}_{2} \mathrm{O}_{5}$ e $\mathrm{K}_{2} \mathrm{O}$, respectivamente; $\mathrm{N} 1 \mathrm{P} 2 \mathrm{~K} 1=70,150$ e $60 \mathrm{~kg}$ ha ${ }^{-1}$ de $\mathrm{N}, \mathrm{P}_{2} \mathrm{O}_{5}$ e $\mathrm{K}_{2} \mathrm{O}$, respectivamente; N1P2K2 = 70, 150 e $120 \mathrm{~kg} \mathrm{ha}^{-1}$ de N, $\mathrm{P}_{2} \mathrm{O}_{5}$ e $\mathrm{K}_{2} \mathrm{O}$, respectivamente; $\mathrm{N} 2 \mathrm{P} 2 \mathrm{~K} 1=140,150$ e $60 \mathrm{~kg} \mathrm{ha}^{-1} \mathrm{de} \mathrm{N}, \mathrm{P}_{2} \mathrm{O}_{5}$ e $\mathrm{K}_{2} \mathrm{O}$, respectivamente. Médias seguidas da mesma letra em cada coluna não diferem pelo teste Scott-Knott a $5 \%$.

Efeitos positivos da aplicação de $\mathrm{P}$ (45 e 90 g/planta de $\mathrm{P}_{2} \mathrm{O}_{5}$ ) no DAC, altura total e fator de produtividade de Pinus taeda, aos seis meses de idade, cultivado em solo Vermelho do norte de Corrientes na Argentina, também foram observados por Fernández et al. (1999). No estudo, os autores também constaram que não houve resposta à aplicação de $\mathrm{K}\left(36\right.$ e $72 \mathrm{~g}$ de $\mathrm{K}_{2} \mathrm{O}$ ) e que ocorria inibição de crescimento à medida que aumentavam a dose de $\mathrm{N}$ (22,5 e $45 \mathrm{~g} /$ planta de $\mathrm{N})$. Vogel et al. (2005) constataram reposta em volume cilíndrico com a adubação no plantio com $\mathrm{P}$ e K (64 e $87 \mathrm{~kg} \mathrm{ha}^{-1}$ de $\mathrm{P}_{2} \mathrm{O}_{5}$ e $\mathrm{K}_{2} \mathrm{O}$, respectivamente), mas não com a aplicação de $\mathrm{N}$, em plantio de Pinus taeda cultivado em um Cambissolo Húmico alumínico típico em Cambará do Sul, RS, aos19 meses de idade.

Para o plantio fertilizado aos cinco anos, os tratamentos N0P1K0, N1P1K1, N1P2K1 e N2P2K1 não diferenciaram estatisticamente entre si, quanto ao ICA de diâmetro das árvores, os quais foram superiores aos demais tratamentos (Quadro 2). Em relação à altura, os tratamentos N1P1K1, N1P2K1 e $\mathrm{N} 2 \mathrm{P} 2 \mathrm{~K} 1$ foram superiores aos demais. Os incrementos, nos respectivos tratamentos, foram de $8,0,12,0$ e $12,0 \%$ no DAP e $8,0,8,0$ e $12 \%$ na $\mathrm{H}$, que consequentemente resultaram em aumentos de 10,1; 14,0; e 15,1 \% no volume de tronco das árvores, em relação à testemunha. Embora o tratamento com aplicação de apenas $\mathrm{P}$ na dose de $60 \mathrm{~kg} \mathrm{ha}{ }^{-1}$ de $\mathrm{P}_{2} \mathrm{O}_{5}$ (N0P1K0) tenha apresentado aumento de $8 \%$ no DAP, o incremento corrente em altura foi $4 \%$ menor que o observado na testemunha, em razão da menor altura observada nesse tratamento. Dessa forma, o incremento apenas em DAP não foi suficiente para promover aumento significativo no incremento em $\mathrm{V}$ das árvores. Os dados obtidos com a floresta fertilizada aos cinco anos de idade evidenciaram que além do $\mathrm{P}$,
$\mathrm{N}$ e K também estão atuando como limitantes no desenvolvimento do Pinus.

No plantio fertilizado aos nove anos, os tratamentos mais eficientes no desenvolvimento das árvores foram o N1P2K1 e N1P2K2, os quais não diferenciaram entre si (Quadro 2). Os respectivos tratamentos promoveram aumentos de 25 e $50 \%$ no ICA-DAP e de 23,5 e 29,4 \% no ICA-H, resultando em ICA-V de 31,1 e 40,6\%. Para os demais tratamentos, as diferentes combinações de fontes e doses de fertilizantes não resultaram em aumento significativo do volume de madeira encontrado para a testemunha. $\mathrm{O}$ tratamento que possui maior dose de $\mathrm{N}(\mathrm{N} 2 \mathrm{P} 2 \mathrm{~K} 1)$ não diferiu da testemunha, e esse efeito pode ser semelhante ao encontrado por Fernandez et al. (1999), os quais relataram que em algumas situações a aplicação de $\mathrm{N}$ tem se apresentado prejudicial ao desenvolvimento das árvores de Pinus.

Os valores de ICA-V médios encontrados nos plantios adubados aos cinco e aos nove anos neste estudo, com médias de 66,9 e $76,6 \mathrm{~m}^{3} \mathrm{ha}^{-1}$, respectivamente, são superiores aos apresentados pela curva de desenvolvimento do ICA do volume de madeira de Pinus taeda, desenvolvida pela Klabin para Otacílio Costa, SC, os quais são 57 e $59 \mathrm{~m}^{3} \mathrm{ha}^{-1}$, respectivamente (Bernett, 2013)(6). Os sítios experimentais adubados, instalados na área de produção de florestas de Pinus da empresa Klabin, produzem volume de tronco além do encontrado nos sítios dessa área. Esses valores também são superiores aos encontrados por Leite et al. (2006), também em Otacílio Costa, SC; porém, sem adubação, sendo de $53,7 \mathrm{~m}^{3} \mathrm{ha}^{-1}$ aos 6,5 anos, e de $64,07 \mathrm{~m}^{3} \mathrm{ha}^{-1}$ aos 10,5 anos. Dacosta (2008) também encontrou valores superiores de 20 a $65 \mathrm{~m}^{3} \mathrm{ha}^{-1}$, aos 6,5 anos, e aos 10,5 anos, de 12 a $38 \mathrm{~m}^{3} \mathrm{ha}^{-1}$, dependendo do sítio. Isso

$\overline{(6)}$ Bernett, 22/04/2013, Klabin S.A., Santa Catarina, Brasil - comunicação pessoal. 
demonstra que os plantios deste experimento estão em boa condição produtiva, acima do relatado pela literatura.

Pelos resultados, pode-se inferir que o melhor efeito da adubação ocorreu sobre o plantio de nove anos, onde os tratamentos N1P2K1 e N1P2K2 proporcionaram aumento médio de aproximadamente $36 \%$ no volume de tronco das plantas, em relação à testemunha. Já o plantio de cinco anos apresentou o menor efeito da aplicação de fertilizante, obtendo incremento médio em torno de13\%, nos tratamentos N1P1K1, N1P2K1 e N2P2K1. Para o plantio de um ano, o aumento foi intermediário, onde todos os tratamentos que receberam fertilizante elevaram o volume de tronco em aproximadamente $26 \%$, em relação à testemunha.

A estimativa do IMA do volume de madeira de Pinus taeda, apresentada na figura 1 , revelou que os tratamentos, que promoveram aumentos significativos no ICA-V, principalmente nos plantios fertilizados com um e nove anos (Quadro 2), proporcionaram efeitos semelhantes no aumento do IMA. No plantio com um ano, todos os tratamentos que receberam adubação (independente do nutriente e dose) aumentaram significativamente o IMA em relação à testemunha, porém não diferenciaram entre si (Figura 1a). Os aumentos nos tratamentos que receberam adubação variaram de 23,0 a $40 \%$, em relação à testemunha.

Para o plantio fertilizado aos cinco anos, diferentemente do que ocorreu no ICA-V (Quadro 2), apenas os tratamentos N1P2K1 e N2P2K1 causaram aumento significativo do IMA em valores próximos a 6,7 e $8,15 \%$, respectivamente, os quais foram estatisticamente superiores aos apresentados pela testemunha e pelos demais tratamentos, que não diferenciaram entre si (Figura 1b). O aumento do ICA$\mathrm{V}$, promovido pela aplicação do tratamento N1P1K1 (70, 75 e $60 \mathrm{~kg} \mathrm{ha}^{-1}$ de $\mathrm{N}, \mathrm{P}_{2} \mathrm{O}_{5}$ e $\mathrm{K}_{2} \mathrm{O}$, respectivamente) não foi suficiente para aumentar significativamente o IMA. Isso pode ter ocorrido em razão do menor desenvolvimento das árvores antes da aplicação da adubação, pois o IMA considera o crescimento da planta desde o plantio, ou seja, a adubação com essas doses promoveu aumento de volume, porém ainda não o suficiente para aumentar o IMA.

O valor médio de IMA para o plantio fertilizado aos cinco anos, obtido neste trabalho, foi de $29,3 \mathrm{~m}^{3} \mathrm{ha}^{-1}$. Dacosta (2008) obteve valores de 12 a $34 \mathrm{~m}^{3} \mathrm{ha}^{-1}$ de IMA, em plantios de mesma idade de avaliação, em condições de diferentes sítios. Em estudo realizado por Leite et al. (2006), os autores verificaram valor máximo para ICA em volume de $14,17 \mathrm{~m}^{3} \mathrm{ha}^{-1}$ para plantios com 6,5 anos. Contudo, em plantios de Pinus taeda sobre um Neossolo Regolítico distroúmbrico léptico em Irati, PR, com diferentes espaçamentos e sem adubação, Lima et al. (2013) encontraram valores de IMA de até $10,65 \mathrm{~m}^{3} \mathrm{ha}^{-1}$ aos quatro anos de idade; já aos sete anos, variaram de 10,68 e $39,27 \mathrm{~m}^{3} \mathrm{ha}^{-1}$.

Em estudo com Pinus taeda, cultivado num Nitossolo Bruno distrófico típico em Capão Alto, SC,
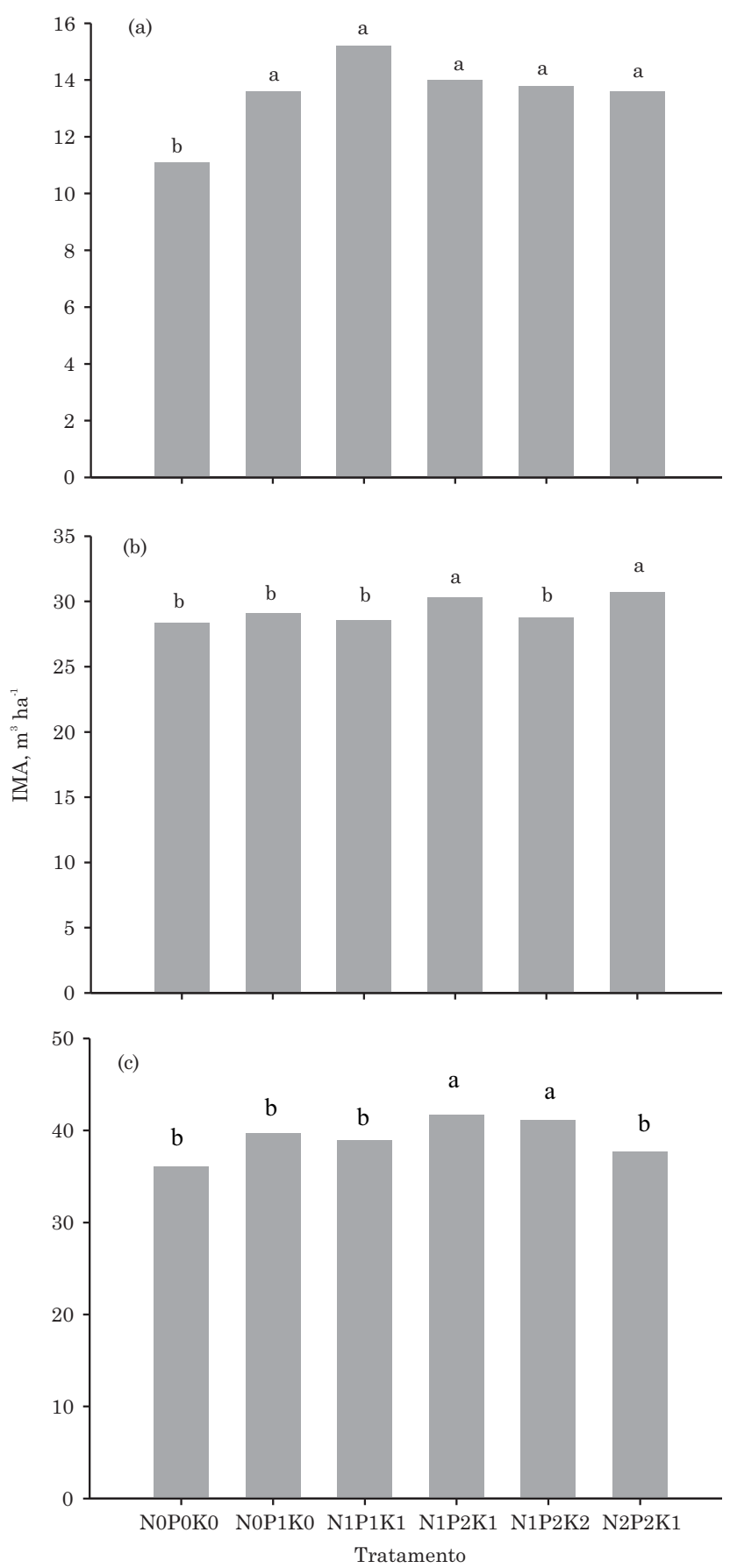

Figura 1. Estimativa do incremento médio anual (IMA) de volume de madeira em florestas de Pinus taeda de um (a), cinco (b) e nove (c) anos, avaliada aos 18 meses após a aplicação dos tratamentos: NOP0K0 $=0 \mathrm{~kg} \mathrm{ha}^{-1}$ de N, $\mathrm{P}_{2} \mathrm{O}_{5} \mathrm{e}$ $\mathrm{K}_{2} \mathrm{O}$; NOP1K0 = 0, 75 e $0 \mathrm{~kg} \mathrm{ha}^{-1}$ de $\mathrm{N}, \mathrm{P}_{2} \mathrm{O}_{5}$ e $\mathrm{K}_{2} \mathrm{O}$, respectivamente; $N 1 P 1 K 1=70,75$ e $60 \mathrm{~kg} \mathrm{ha}^{-1}$ de $\mathrm{N}, \mathrm{P}_{2} \mathrm{O}_{5}$ e $\mathrm{K}_{2} \mathrm{O}$, respectivamente; $\mathrm{N} 1 \mathrm{P} 2 \mathrm{~K} 1=$ 70,150 e $60 \mathrm{~kg} \mathrm{ha}^{-1}$ de $\mathrm{N}, \mathrm{P}_{2} \mathrm{O}_{5}$ e $\mathrm{K}_{2} \mathrm{O}$, respectivamente; $\mathrm{N} 1 \mathrm{P} 2 \mathrm{~K} 2=70,150$ e $120 \mathrm{~kg} \mathrm{ha}^{-1}$ de $\mathrm{N}, \mathrm{P}_{2} \mathrm{O}_{5}$ e $\mathrm{K}_{2} \mathrm{O}$, respectivamente; $\mathrm{N} 2 \mathrm{P} 2 \mathrm{~K} 1=$ 140,150 e $60 \mathrm{~kg} \mathrm{ha}^{-1}$ de $\mathrm{N}, \mathrm{P}_{2} \mathrm{O}_{5}$ e $\mathrm{K}_{2} \mathrm{O}$, respectivamente. Médias seguidas da mesma letra não diferem pelo teste Scott-Knott a $5 \%$. 
Simonete et al. (2011) verificaram que aplicação de adubação no plantio com doses partir de $50 \mathrm{~kg} \mathrm{ha}^{-1}$ de $\mathrm{P}_{2} \mathrm{O}_{5}$, utilizando-se a formulação 6:30:6 $\left(\mathrm{N}: \mathrm{P}_{2} \mathrm{O}_{5}: \mathrm{K}_{2} \mathrm{O}\right)$, promoveu incremento significativo de $13 \%$ no volume de madeira do Pinus, aos cinco anos de idade, em relação à aplicação da mesma dose de $\mathrm{P}_{2} \mathrm{O}_{5}$ na forma de superfosfato triplo e testemunha; valor superior ao encontrado neste experimento no plantio fertilizado aos cinco anos, em que o maior incremento no IMA foi de $8,1 \%$ na dosagem N2P2K1, sem se diferenciar do N1P2K1, com 6,7 \%.

No plantio fertilizado aos nove anos, somente os tratamentos N1P2K1 e N1P2K2 promoveram incrementos significativos no IMA, 15,5 e 13,9 \%, respectivamente, em relação à testemunha, mas não apresentaram diferença entre si (Figura 1c). O valor de IMA para o plantio de nove anos obtido neste estudo foi de $39,2 \mathrm{~m}^{3} \mathrm{ha}^{-1}$, o qual se encontra próximo à média dos valores mais altos, ou até mesmo acima da média dos valores citados na literatura. Valores de 14 a 42 $\mathrm{m}^{3} \mathrm{ha}^{-1}$ de IMA, em diferentes sítios de plantios com Pinus taeda com mesma idade de avaliação, foram encontrados por Dacosta (2008). Já Leite et al. (2006) encontraram valor médio de $30,18 \mathrm{~m}^{3} \mathrm{ha}^{-1}$ para Pinus taeda, com 9,5 anos de idade.

Os valores de IMA nos plantios adubados aos cinco e aos nove anos, 29,3 e $39,2 \mathrm{~m}^{3} \mathrm{ha}^{-1}$ respectivamente, observados neste estudo foram superiores aos apresentados na curva de desenvolvimento do IMA do volume de madeira de Pinus taeda, desenvolvida pela Klabin para região de Otacílio Costa, SC, os quais correspondem a 26 e $36 \mathrm{~m}^{3} \mathrm{ha}^{-1}$, respectivamente (Bernett, 2013) ${ }^{(6)}$. Desse modo, a adoção da prática de fertilização em plantios comerciais da empresa Klabin pode estar promovendo aumento do IMA de volume de madeira de Pinus, com perspectivas de maior produtividade e rentabilidade futura, principalmente em sítios de plantios compatíveis ao do presente estudo.

Quanto ao estado nutricional dos plantios, em geral, não houve efeito significativo da aplicação dos tratamentos nos teores de N, P e K nas acículas dos três plantios de Pinus, nos dois períodos de avaliação (Quadro 3), exceto para o plantio de cinco anos, onde os teores de K nos tratamentos N1P2K1, N1P2K2 e $\mathrm{N} 2 \mathrm{P} 2 \mathrm{~K} 1$, aos seis meses de avaliação, foram inferiores aos demais tratamentos, bem como os teores de $\mathrm{P}$, nos tratamentos N1P1K1, N1P2K1 e N1P2K2, aos 18 meses de avaliação, foram superiores. A ausência de alteração no teor foliar de $\mathrm{N}$ e $\mathrm{P}$ em plantio de Pinus taeda, cultivado num Argissolo Vermelho em Montecarlo, Misiones, Argentina, fertilizado com 46 e $96 \mathrm{~g} /$ planta de $\mathrm{N}$ e $\mathrm{P}_{2} \mathrm{O}_{5}$, respectivamente, também foi constada por Faustino et al. (2013).

Os resultados para os teores de N, P e K, obtidos neste estudo, podem estar associados à maior diluição dos elementos na biomassa produzida em maior

Quadro 3. Teores médios de N, P e K nas acículas em florestas de Pinus taeda com idades de um, cinco e nove anos, avaliadas aos seis e 18 meses após a aplicação dos tratamentos

\begin{tabular}{|c|c|c|c|c|c|c|c|c|c|}
\hline \multirow{2}{*}{ Tratamento $^{(1)}$} & \multicolumn{3}{|c|}{ Pinus de um ano } & \multicolumn{3}{|c|}{ Pinus de cinco anos } & \multicolumn{3}{|c|}{ Pinus de nove anos } \\
\hline & $\mathbf{N}$ & $\mathbf{P}$ & $\mathbf{K}$ & $\mathbf{N}$ & $\mathbf{P}$ & $\mathbf{K}$ & $\mathbf{N}$ & $\mathbf{P}$ & $\mathbf{K}$ \\
\hline & & & & & 6 meses & & & & \\
\hline N0P0K0 & $1,24 \mathrm{a}$ & $0,15 \mathrm{a}$ & $0,75 \mathrm{a}$ & $1,24 \mathrm{a}$ & $0,24 \mathrm{a}$ & $0,80 \mathrm{a}$ & $1,20 \mathrm{a}$ & $0,10 \mathrm{a}$ & $0,63 \mathrm{a}$ \\
\hline N0P1K0 & $1,37 \mathrm{a}$ & $0,15 \mathrm{a}$ & $0,89 \mathrm{a}$ & $1,17 \mathrm{a}$ & $0,19 \mathrm{a}$ & $0,76 \mathrm{a}$ & $1,32 \mathrm{a}$ & $0,17 \mathrm{a}$ & $0,64 \mathrm{a}$ \\
\hline N1P1K1 & $1,30 \mathrm{a}$ & $0,22 \mathrm{a}$ & $0,87 \mathrm{a}$ & $1,26 \mathrm{a}$ & $0,29 \mathrm{a}$ & $0,76 \mathrm{a}$ & $1,28 \mathrm{a}$ & $0,19 \mathrm{a}$ & $0,75 \mathrm{a}$ \\
\hline N1P2K1 & $1,31 \mathrm{a}$ & $0,23 \mathrm{a}$ & $0,86 \mathrm{a}$ & $1,30 \mathrm{a}$ & $0,29 \mathrm{a}$ & $0,73 \mathrm{~b}$ & $1,55 \mathrm{a}$ & $0,25 \mathrm{a}$ & $0,60 \mathrm{a}$ \\
\hline N1P2K2 & $1,27 \mathrm{a}$ & $0,20 \mathrm{a}$ & $0,73 \mathrm{a}$ & $1,13 \mathrm{a}$ & $0,18 \mathrm{a}$ & $0,68 \mathrm{~b}$ & $1,26 \mathrm{a}$ & $0,13 \mathrm{a}$ & $0,71 \mathrm{a}$ \\
\hline N2P2K1 & $1,38 \mathrm{a}$ & $0,25 \mathrm{a}$ & $0,92 \mathrm{a}$ & $1,20 \mathrm{a}$ & $0,13 \mathrm{a}$ & $0,67 \mathrm{~b}$ & $1,34 \mathrm{a}$ & $0,15 \mathrm{a}$ & $0,57 \mathrm{a}$ \\
\hline \multirow[t]{2}{*}{ CV (\%) } & 6,6 & 31,7 & 12,7 & 4,7 & 33,6 & 4,3 & 12,8 & 49,6 & 24,7 \\
\hline & \multicolumn{9}{|c|}{18 meses } \\
\hline N0P0K0 & $1,47 \mathrm{a}$ & $0,12 \mathrm{a}$ & $0,49 \mathrm{a}$ & $1,63 \mathrm{a}$ & $0,14 \mathrm{~b}$ & $0,52 \mathrm{a}$ & $1,73 \mathrm{a}$ & $0,15 \mathrm{a}$ & $0,54 \mathrm{a}$ \\
\hline N0P1K0 & $1,43 \mathrm{a}$ & $0,14 \mathrm{a}$ & $0,52 \mathrm{a}$ & $1,60 \mathrm{a}$ & $0,14 \mathrm{~b}$ & $0,49 \mathrm{a}$ & $1,73 \mathrm{a}$ & $0,17 \mathrm{a}$ & $0,54 \mathrm{a}$ \\
\hline N1P1K1 & $1,50 \mathrm{a}$ & $0,13 \mathrm{a}$ & $0,50 \mathrm{a}$ & $1,70 \mathrm{a}$ & $0,16 \mathrm{a}$ & $0,59 \mathrm{a}$ & $1,75 \mathrm{a}$ & $0,15 \mathrm{a}$ & $0,53 \mathrm{a}$ \\
\hline N1P2K1 & $1,47 \mathrm{a}$ & $0,14 \mathrm{a}$ & $0,51 \mathrm{a}$ & $1,57 \mathrm{a}$ & $0,15 \mathrm{a}$ & $0,54 \mathrm{a}$ & $1,70 \mathrm{a}$ & $0,16 \mathrm{a}$ & $0,50 \mathrm{a}$ \\
\hline N1P2K2 & $1,43 \mathrm{a}$ & $0,14 \mathrm{a}$ & $0,52 \mathrm{a}$ & $1,73 \mathrm{a}$ & $0,16 \mathrm{a}$ & $0,56 \mathrm{a}$ & $1,70 \mathrm{a}$ & $0,16 \mathrm{a}$ & $0,58 \mathrm{a}$ \\
\hline N2P2K1 & $1,43 \mathrm{a}$ & $0,13 \mathrm{a}$ & $0,50 \mathrm{a}$ & $1,67 \mathrm{a}$ & $0,14 \mathrm{~b}$ & $0,53 \mathrm{a}$ & $1,70 \mathrm{a}$ & $0,16 \mathrm{a}$ & $0,52 \mathrm{a}$ \\
\hline CV (\%) & 6,1 & 8,9 & 10,3 & 8,0 & 6,4 & 9,7 & 5,2 & 6,6 & 8,0 \\
\hline
\end{tabular}

(1) $\mathrm{N} 0 \mathrm{P} 0 \mathrm{~K} 0=0 \mathrm{~kg} \mathrm{ha}^{-1}$ de $\mathrm{N}, \mathrm{P}_{2} \mathrm{O}_{5}$ e $\mathrm{K}_{2} \mathrm{O} ; \mathrm{N} 0 \mathrm{P} 1 \mathrm{~K} 0=0,75$ e $0 \mathrm{~kg} \mathrm{ha}^{-1}$ de $\mathrm{N}, \mathrm{P}_{2} \mathrm{O}_{5}$ e $\mathrm{K}_{2} \mathrm{O}$, respectivamente; N1P1K1 = 70, 75 e $60 \mathrm{~kg} \mathrm{ha}^{-1}$ de N, $\mathrm{P}_{2} \mathrm{O}_{5}$ e $\mathrm{K}_{2} \mathrm{O}$, respectivamente; $\mathrm{N} 1 \mathrm{P} 2 \mathrm{~K} 1=70,150$ e $60 \mathrm{~kg}$ ha ${ }^{-1}$ de N, $\mathrm{P}_{2} \mathrm{O}_{5}$ e $\mathrm{K}_{2} \mathrm{O}$, respectivamente; N1P2K2=70, 150 e $120 \mathrm{~kg} \mathrm{ha}^{-1}$ de $\mathrm{N}, \mathrm{P}_{2} \mathrm{O}_{5}$ e $\mathrm{K}_{2} \mathrm{O}$, respectivamente; $\mathrm{N} 2 \mathrm{P} 2 \mathrm{~K} 1=140,150$ e $60 \mathrm{~kg} \mathrm{ha}^{-1}$ de $\mathrm{N}, \mathrm{P}_{2} \mathrm{O}_{5}$ e $\mathrm{K}_{2} \mathrm{O}$, respectivamente. Médias seguidas da mesma letra em cada coluna não diferem pelo teste Scott-Knott a $5 \%$. 
quantidade pelas árvores de Pinus, que receberam fertilização, e à capacidade das árvores em manter certo equilíbrio entre os teores desses nutrientes nas folhas, mesmo havendo maior disponibilidade dos elementos no solo. Contudo, os teores observados em todos os tratamentos encontram-se dentro da faixa de disponibilidade considerada adequada para a cultura, sugerida pela CQFSRS/SC (2004) e por Gonçalves (1995).

\section{CONCLUSÕES}

1. O Pinus taeda apresentou incremento no volume de madeira em respostas à adubação NPK em todas as idades avaliadas.

2. O maior incremento de volume de tronco foi obtido no plantio de nove anos, com a adição de doses de $70 \mathrm{~kg} \mathrm{ha}^{-1}$ de $\mathrm{N}, 75 \mathrm{~kg} \mathrm{ha}^{-1}$ de $_{2} \mathrm{O}_{5}$ e $60 \mathrm{~kg} \mathrm{ha}^{-1}$ de $\mathrm{K}_{2} \mathrm{O}$, seguido do plantio de um ano, onde o incremento máximo foi atingido com aplicação de $70 \mathrm{~kg} \mathrm{ha}^{-1} \mathrm{de}$ $\mathrm{P}_{2} \mathrm{O}_{5}$.

3. O plantio de cinco anos apresentou o menor efeito da aplicação de fertilizante, obtendo incremento máximo com a adição de doses de $70 \mathrm{~kg} \mathrm{ha}^{-1}$ de $\mathrm{N}$, $75 \mathrm{~kg} \mathrm{ha}^{-1}$ de $\mathrm{P}_{2} \mathrm{O}_{5}$ e $60 \mathrm{~kg} \mathrm{ha}^{-1}$ de $\mathrm{K}_{2} \mathrm{O}$.

\section{LITERATURA CITADA}

ALBAUGH, T.J., ALLEN, H.L. \& FOX; T.R. Historical patterns of forest fertilization in the southeastern United States from 1969 to 2004. J. Appl. For., 31:129-137, 2007.

ALBAUGH, T.J., ALLEN, H.L. \& FOX, T.R. Nutrient use and uptake in Pinus taeda. Tree Physiol., 28:1083-1098, 2008.

BEKELE, A.; HUDNALL, W.H. \& TIARKS, A.E. Response of densely stocked loblolly pine (Pinus taeda L.) to applied nitrogen and phosphorus. J. Appl. For., 27:180189, 2003.

CLARK III, A. \& SAUCIER, J. R. Influence of planting density, intensive culture, geographic location, and species on juvenile wood formation in Southern Pine. Research Division Georgia Forest Commission, 1991. 14p. (Technical Paper 85)

COMISSÃO DE QUÍMICA E FERTILIDADE DO SOLO RS/SC - CQFSRS/SC. Manual de adubação e de calagem para o Estado do Rio Grande do Sul e Santa Catarina. Porto Alegre, SBCS/Núcleo Regional Sul, UFRGS, 2004. 400p.

DACOSTA, L.P.E. Relações biométricas em povoamentos jovens de Pinus taeda L. na Província de Corrientes, República Argentina. Santa Maria, Universidade Federal de Santa Maria, 2008. 128p. (Tese de Doutorado)

ENCINAS, J.I.; SILVA, G.F. \& PINTO, J.R.R. Idade e crescimento das árvores. Brasília, Comunicações Técnicas Florestais, UnB, 2005. 47p.
EVERETT, C.J. \& PALM-LEIS, H. Availability of residual phosphorus fertilizer for loblolly pine. For. Ecol. Manage., 258:2207-2213, 2009.

FAUSTINO, L.I.; BULFE, N.; PINAZO, M.; GOYA, J.F.; MARTIARENA, R.; KNEBEL. O. \& GRACIANO, C. Pinus taeda $\mathrm{L}$. initial growth in response to $\mathrm{N}$ and $\mathrm{P}$ fertilization, on a stony soil of Misiones province. Rev. For. Yvyraretá, 18:52-57, 2011.

FAUSTINO, L.I.; BULFE, N.M.; PINAZO, M.A.; MONTEOLIVA, S.E. \& GRACIANO, C. Dry weight partitioning and hydraulic traits in young Pinus taeda trees fertilized with nitrogen and phosphorus in a subtropical area. Tree Physiol., 33:241-251, 2013.

FERREIRA, C.A.; SILVA, H.D.; REISSAMNN, C.B.; BELLOTE, A.F.J. \& MARQUES, R. Nutrição de Pinus no sul do Brasil - diagnóstico e propriedades de pesquisa. Colombo, Embrapa Florestas, 2001. 23p. (Documentos, 60)

FERNÁNDEZ, R.; ASPILLAGA, F.R.; LUPI, A.; HERNANDEZ, A. \& REIS, H. Efectos de diferentes prácticas de preparación del terreno y fertilización sobre el crecimiento inicial del Pinus spp en el NE argentino. Bosque, 20:47-52, 1999.

FERNÁNDEZ, R.; ASPILLAGA, F.R.; LUPI, A.; LOPEZ, E.; PEZZUTTI, R.V.; CRECHI, E.; PAHR, N.; NATIUCK, M. \& CORTEZ, M. Respuesta del Pinus taeda y la Araucaria angustifolia a la adición de N, P y K em la implantación. In: SILVOARGENTINA, 1., 2000, Governador Virasoro. Anais... Governador Virasoro, Corrientes, 2000. CD-ROM

FLINN, D.W. Practical aspects of the nutrition of exotic conifer plantations and native eucalypt forests in Australia. MelBourne, Research for Forest Management/CSIRO, 1985. 296p.

FOELKEL, C.E.B. Celulose kraft de Pinus spp. O Papel, 18:4967, 1976.

FOX, T.R.; ALLEN, H.L.; ALBAUGH, T.J.; RUBILAR, R.A. \& CARLSON, C. Tree nutrition and forest fertilization of pine plantations in the southern United States. South. J. Appl. For. 31:5-11, 2007.

GONÇALVES, J.L.M. Recomendações de adubação para Eucalyptus, Pinus e espécies típicas da Mata Atlântica. Doc. Flor., 15:1-23, 1995.

IBAÑEZ, C.; NUÑEZ, P.; PEZZUTTI, R. \& RODRIGUEZ, F. Efectos de la roturación del suelo y fertilización con fósforo en el crecimiento inicial de plantaciones de Pinus taeda, en suelos rojos del Noreste de la provincia de Corrientes, Argentina. Bosque, 25:69-77, 2004.

JOKELA, E.J.; ALLEN, H.L. \& McFEE, W.W. Fertilization of southern pines at establishment. In: DURYEA, M. \& DOUGHERTY, P., eds. Forest regeneration manual. London, Kluwer Academic Publishers, 1991. p.263-277.

JOKELA, E.J. \& LONG, A.J. Using soils to guide fertilizer recommendations for southern pines. Gainesville, University of Florida/Institute of Food an Agricultural Sciences Cooperative Extension, 2000. 11p. (Circular 1230)

JOKELA, E.J. Nutrient management of southern pines. In: DICKENS, E.D.; BARNETT, J.P.; HUBBARD, W.G. \& JOKELA, E.J., eds. Slash pine: Still growing and growing. Proceeding of the slash pine symposium. Washington, USDA/Forest Service General, 2004. p.27-35. (Technical Report SRS-76) 
JUVENAL, T.L. \& MATTOS, R.L.G. O setor florestal no Brasil e a importância do reflorestamento. BNDES Setorial, 16:330, 2002 .

KYLE, K.H.; ANDREWS, L.J.; FOX, T.R.; AUST, M.; BURGER, J.A. \& HANSEN, G.H. Long-term effects of drainage, bedding, and fertilization on growth of loblolly pine (Pinus taeda L.) in the coastal plain of Virginia. South. J. Appl. For., 29:205-214, 2005.

LEITE, H.G.; NOGUEIRA, G.S. \& MOREIRA, A.M. Efeito do espaçamento e da idade sobre variáveis de povoamentos de Pinus taeda L. R. Árvore, 30:603-613, 2006.

LIMA, R.; INOUE, M.T.; FIGUEIREDO FILHO, A.; ARAUJO, A.J. \& MACHADO, S.A. Efeito do espaçamento no desenvolvimento volumétrico de Pinus taeda L. Flor. Amb., 20:223-230, 2013.

MURPHY, J. \& RILEY, J.P. A modified single solution method for the determination of phosphate in natural waters. Anal. Chim. Acta, 27:31-36, 1962.

PINTO JUNIOR, J.E.; SANTOS, P.E.T.; AGUIAR, A.V.; KALIL FILHO, A.N.; PALUDZYSZYN FILHO, E.; STURION, J.A.; RESENDE, M.D.V. \& SOUSA, V.A. Melhoramento genético de espécies arbóreas na Embrapa Florestas: uma visão histórica. Colombo: Embrapa Florestas, 2013. 109 p. (Embrapa Florestas. Documentos, 259)

R DEVELOPMENT CORE TEAM. R: A language and environment for statistical computing. Vienna, R Foundation for Statistical Computing/The $R$ Foundation for Statistical Computing. ISBN: 3-900051-07-0. 2011. Disponível em: <http://www.R-project.org/>.
REISSMANN, C.B. \& WISNIEWSKI, C. Aspectos nutricionais de plantios de Pinus. In: GONÇALVES, J.L.M. \& BENEDETTI, V., eds. Nutrição e fertilização florestal. 2.ed. Piracicaba, IPEF, 2005. p.135-166.

SANTA CATARINA. Otacílio Costa. Disponível em: <http:// w w w. s c. g o v. b r / p o r t a l t u r i s m o / Default.asp? CodMunicipio $=285 \&$ Pag $=1>$. Acesso em 26 ago. 2012.

SIMONETE, M.A.; MORO, L.; CHAVES, D.M. \& TEIXEIRA, C.F.A. Efeito da adubação de plantio em Pinus taeda L. aos cinco anos de idade. In: CONGRESSO BRASILEIRO DE CIÊNCIA DO SOLO, 33., Uberlândia, 2011. Anais... Uberlândia, Universidade Federal de Uberlândia, 2011. CD-ROM

TEDESCO, M.J.; GIANELLO, C.; BISSANI, C.; BOHNEN, H. \& VOLKWEISS, S.J. Análise de solo, plantas e outros materiais. 2.ed. Porto Alegre, Universidade Federal do Rio Grande do Sul, 1995. 174p. (Boletim Técnico, 5)

VIERA, M. \& SCHUMACHER, M.V. Concentração e retranslocação de nutrientes em acículas de Pinus taeda L. Ci. Flor.,19:375-382, 2009.

VOGEL, H.L.M.; SCHUMACHER, M.V.; STORCK, L. \& WITSCHORECK, R. Crescimento inicial de Pinus taeda L. relacionado a doses de N, P e K. Ci. Flor., 15:199-206, 2005.

WILL, R.E.; MARKEWITZ, D.; HENDRICK, R.; MEASON, D.F.; CROCKER, T.R. \& BORDERS, B.E. Nitrogen and phosphorus dynamics for 13-year-old loblolly pine stands receiving complete competition control and annual $\mathrm{N}$ fertilizer. For. Ecol. Manage., 227:155-168, 2006. 